\title{
Aspectos pedagógicos, tecnológicos y de interacción social del aprendizaje móvil: revisión Sistemática de Literatura
}

\section{Pedagogical, technological and social interaction aspects of mobile learning: A systematic review}

\author{
Alicia González Pérez ${ }^{1}$ \\ aliciagp@unex.es \\ Maria José Sosa Díaz \\ mjosesosa@unex.es \\ Universidad de Extremadura, Cáceres, España
}

\section{Resumen:}

El uso de tecnologías móviles se incrementa de forma exponencial en contextos formativos, favoreciendo metodologías de enseñanza- aprendizaje más dinámicas, flexibles y abiertas. Con el objetivo de elaborar un modelo explicativo en el que se identifiquen los elementos más significativos que emergen del mobile learning, se ha realizado una revisión sistemática de artículos científicos indexados en bases de datos del área de Educación, ERIC y Dialnet, considerando varios criterios de búsqueda. Posteriormente, se analizaron los artículos seleccionados para la conformación de un modelo explicativo del mobile learning considerando tres aspectos: pedagógicos, tecnológicos y de interacción social. Finalmente, se abre un apartado de discusión donde se dan claves para repensar la Educación que queremos para una sociedad cada vez más tecnologizada y se pone en valor algunos de los retos para la Educación del futuro: aprendizaje

\begin{abstract}
:
The use of mobile technologies is increasing exponentially in training contexts as a way to enable dynamic, flexible and open teaching-learning methodologies. This article presents a systematic review carried out considering scientific articles indexed in the Education databases ERIC and Dialnet. We aimed to develop an explanatory model in which elements emerging from mobile learning were identified. Articles were analyzed to configure an explanatory mobile learning model considering three aspects: pedagogical, technological and social interaction. The results show key elements that help to understand the importance of mobile learning in designing a student-centered learning process that addresses the needs of collaboration and interrelationship between users both in social media environments and outside the classroom. It also emphasizes the need for mobile learning to be connected, to be open, flexible, ubiquitous, and
\end{abstract}

1 Dirección para correspondencia (correspondence address):

Alicia González Pérez. Departamento de Ciencias de la Educación. Universidad de Extremadura. Avd. de la Universidad s/n, 10003 - Cáceres (España). 
Aspectos pedagógicos, tecnológicos y de interacción social del aprendizaje móvil: revisión Sistemática de Literatura

Alicia González Pérez y Maria José Sosa Díaz

móvil, conectado y autoregulado por el estudiante, aprendizaje abierto, flexible y ubicuo, y la implementación de metodologías activas de aprendizaje.

\section{Palabras clave:}

Aprendizaje móvil; métodos de enseñanza; Tecnologías de la Información y la Comunicación (TIC); aprendizaje autoregulado; aprendizaje colaborativo. implemented through active learning methodologies.

\section{Key words:}

Mobile learning; Teaching methods; Information and Communication Technologies (ICT); Self-regulated learning; Collaborative learning.

\section{Résumé:}

L'utilisation des technologies mobiles augmente de manière exponentielle dans les contextes de formation, favorisant des méthodologies d'enseignement-apprentissage plus dynamiques, flexibles et ouvertes. Dans le but de développer un modèle explicatif dans lequel les éléments les plus significatifs du mobilelearning sont identifiés, une revue systématique d'articles scientifiques indexés dans des bases de données du secteur d'Éducation, ERIC et Dialnet, prenant en compte plusieurs critères de recherche, a été réalisée. Par la suite, les articles sélectionnés ont été analysés pour la configuration d'un modèle explicatif du mobilelearning en considérant trois aspects: I'interaction pédagogique, technologique et sociale. Les résultats montrent des preuves et des éléments clés qui aident à comprendre l'importance de l'apprentissage mobile dans la conception d'un processus d'apprentissage centré sur l'élève, qui répond aux besoins de collaboration et d'interrelation entre utilisateurs dans des contextes sociaux en ligne et en dehors de la salle de classe. II met également l'accent sur la nécessité pour l'apprentissage mobile d'être connecté, ouvert, flexible, omniprésent et mis en œuvre par le biais de méthodologies d'apprentissage actif.

\section{Mots clés:}

Apprentissage mobile méthodes d'enseignement Technologies de l'information et de la communication (TIC); apprentissage autorégulé; apprentissage collaboratif.

Fecha de recepción: 10-10-2018

Fecha de aceptación: 19-12-2019

\section{Introducción}

Hay que tener en cuenta que el uso global de Internet se ha visto incrementado en un 362,3\% entre el 2000 y el 2009 (Rannu, Saksing, \& Mahlakõiv, 2010). Este uso continúa incrementándose debido al aumento exponencial de dispositivos móviles (smartphones, PDAs, laptops, tablets, entre otros) en todo el mundo. Internet es usado y conocido en casi todas las culturas del mundo y está siendo incorporado en todos los aspectos fundamentales del día a día. Es por ello que el profesorado de todos los niveles no debe permanecer al margen de esta tendencia y tiene la responsabilidad de explorar la naturaleza ubicua de las tecnologías 
móviles para mejorar los procesos de enseñanza-aprendizaje, y ver su impacto en los estudiantes.

Dos cuestiones importantes que no deben pasar desapercibidas en una sociedad tecnologizada son, por un lado, la demanda social es la que impone los cambios (Tedesco, 2000), y por otro lado, los estudiantes de hoy, que evolucionan al ritmo de las tecnologías, las integran progresivamente en sus procesos de enseñanza-aprendizaje. De ahí que es interesante considerar a los estudiantes como usuarios potenciales tanto de los aprendizajes móviles, ya que son parte de su proceso de enseñanza/aprendizaje, como de dispositivos móviles tales como smartphones, PDAs, laptops y tablets, entre otras. Es por ello que la necesidad de incluir estas herramientas en las aulas se ha convertido en un tema de especial interés en el campo de la Educación (Godejord, González-Pérez, Figueiredo, \& Solmaz, 2016).

Sin duda, no se puede obviar que las tecnologías móviles tienen el potencial de permitir, a los estudiantes, profesores y al personal de administración y servicios, el acceso y uso de los servicios móviles, desde cualquier lugar y en cualquier momento.

Es en este contexto donde emerge el concepto de mobile learning como una evolución del e-learning, al que se le incorporan distintos usos educativos (Althunibat, 2015). Pero además borra las barreras temporales, y ofrece la posibilidad de conectarse en cualquier momento y lugar (Gómez \& Monge, 2013). Así pues, el mobile learning favorece la obtención de materiales y recursos para el aprendizaje, y facilita la realización de actividades y tareas desde cualquier momento o lugar (Reychav \& Wu, 2015). Mientras el e-learning lleva a los estudiantes más allá del aula tradicional, el mobile learning los lleva más allá del aula y fuera de una ubicación fija (Brantes et al., 2013).

Algunos cambios asociados al mobile learning en el ámbito educativo también van en la línea de ofrecer un aprendizaje personalizado, colaborativo e informal en diferentes contextos. Soykan \& Uzunboylu (2015) identifican algunas ventajas del uso de los dispositivos móviles en el sentido de favorecer el aprendizaje permanente y el aprendizaje autónomo e invisible, adaptado a las necesidades inmediatas, independiente del momento y la ubicación. Yot \& Marcelo (2015) resaltan que los smartphones tienden a ser más utilizados para aprendizajes que se dan al aire libre, en espacios abiertos, mientras se viaja y en establecimientos públicos. Sin embargo, las tablets se usan más en el hogar compartiendo 
Aspectos pedagógicos, tecnológicos y de interacción social del aprendizaje móvil: revisión Sistemática de Literatura

Alicia González Pérez y Maria José Sosa Díaz

su presencia con el ordenador portátil. Según los resultados obtenidos en cuanto al uso de los dispositivos móviles en los procesos formativos formales, Yot \& Marcelo (2015) concluyen que el nivel de uso de los dispositivos móviles es muy bajo o casi inexistente en contextos formativos formales, a pesar de las posibilidades educativas que ofrece.

No obstante, existen algunas experiencias en Educación donde se integran las Tecnologías de la Información y Comunicación (TIC), promocionando el uso de los dispositivos móviles dentro del aula (Kong \& Song, 2015). Bring Your Own Device (BYOD) es una iniciativa que tiene como objetivo que los estudiantes Ileven su ordenador personal al centro y lo utilicen como herramienta para el aprendizaje. Esta iniciativa favorece el uso del dispositivo como herramienta de aprendizaje al disponer de ella en cualquier momento o lugar (Kong \& Song, 2015; Reychav \& Wu, 2015).

Otras iniciativas formativas están relacionadas con el uso e introducción de aplicaciones móviles (Apps) en el aula. Este fenómeno ha sido ampliamente tratado por la comunidad científica a través de numerosas investigaciones que se van a mostrar a lo largo del artículo (Adegbija \& Bola, 2015; Alhassan, 2016; Al-said, 2015; Althunibat, 2015; Álvarez \& Gisbert, 2015; Antunes \& Tenorio, 2010; Bahry, Anwar, Amran, \& Rias, 2015; Brantes et al., 2013; Cheon, Lee, Crooks, \& Song, 2012; Gikas \& Grant, 2013; Goh, Quek, \& Lee, 2010; Joan, 2013; Kong \& Song, 2015; Nakano Osores et al., 2013; Organista \& Serrano, 2011; Reychav \& Wu, 2015; Sarrab, Elbasir, \& Alnaeli, 2016; Su \& Cheng, 2013; Suárez, Crescenzi, \& Grané, 2013; Tabuenca, Kalz, Drachsler, \& Specht, 2015; Wang, Shannon, \& Ross, 2013; Yot \& Marcelo, 2015).

\section{Metodología}

Considerar a los estudiantes del futuro como usuarios potenciales de aprendizajes móviles es una realidad y por ello se considera importante identificar los elementos más significativos que emergen del mobile learning, para entender mejor la complejidad de esta realidad. Para ello, se ha desarrollado una revisión sistemática de literatura de artículos científicos indexados en bases de datos del área de educación como son ERIC y Dialnet.

Según Kitchenham (2004, p.1) la revisión sistemática de bibliografía 
es "un método que permite la evaluación e interpretación de todas las investigaciones a nuestro alcance que resulten relevantes para una pregunta de investigación, tema o evento de interés". Teniendo en cuenta esta definición, la revisión sistemática supone el mejor método para:

- Identificar los aspectos más relevantes sobre el aprendizaje a través de dispositivos móviles para comprender mejor el término de mobile learning.

- Realizar una reflexión crítica de los elementos que se apuntan en los diferentes estudios sobre mobile learning y su incidencia en el aprendizaje.

Para la realización de la revisión, se ha llevado a cabo un proceso estructurado de búsqueda de artículos científicos. Esta revisión bibliográfica se ha abordado desde una perspectiva cualitativa, con carácter exploratorio, inductivo y descriptivo, para poder conocer, describir, comprender e interpretar los elementos más significativos del mobile learning. Como puede observarse en la figura 1, este proceso se dividió en tres etapas diferenciadas (Gómez, Fernando, Aponte, \& Betancourt, 2014; Guirao, Olmedo, \& Ferrer, 2008; Kitchenham, 2004). En primer lugar, una etapa de diseño del protocolo de búsqueda de la información, en el que se define el objetivo y las preguntas de investigación, se establece el protocolo de búsqueda en las bases de datos y los criterios de selección. En segundo lugar, se procedió a la consulta de las bases de datos y recopilación de los estudios de investigación que cumplieran los criterios acordados, para extraer la información más relevante sobre el mobile learning. Y por último, se realizó el análisis y organización de la información encontrada de manera estructurada para identificar los elementos clave que dinamizan el mobile learning.
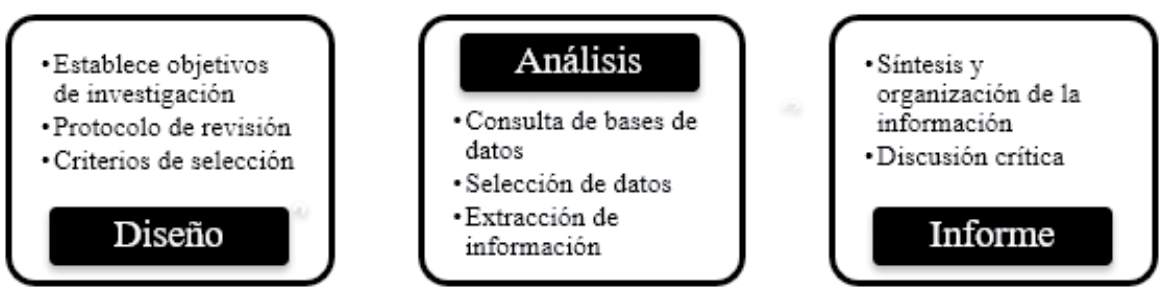

Figura 1. Etapas de revisión sistemática de la bibliografía (Elaboración propia) 
Aspectos pedagógicos, tecnológicos y de interacción social del aprendizaje móvil: revisión Sistemática de Literatura

Alicia González Pérez y Maria José Sosa Díaz

Aunque las etapas identificadas pueden parecer secuenciales, lo cierto es que todas ellas han implicado iteración, ya que, algunas de ellas han sido refinadas o concluidas en una etapa posterior del protocolo marcado (Kitchenham, 2004).

\subsection{Diseño y planificación}

Con el fin de examinar el estado de la cuestión del mobile learning e identificar las dimensiones claves que inciden en el aprendizaje móvil en las distintas etapas educativas, se plantean las siguientes preguntas de investigación:

Pregunta 1: ¿Cuáles son las dimensiones que definen el mobile learning y qué elementos dinamizan el proceso de aprendizaje a través de dispositivos móviles?

Pregunta 2: ¿Qué tipos de aprendizaje se producen a través de dispositivos móviles según la literatura?

Para dar respuestas a estas preguntas se delimitan cuatro criterios a considerar durante la búsqueda bibliográfica. Éstos son:

Tabla 1

Criterios de inclusión - exclusión

\begin{tabular}{lll}
\hline Categoría & Descripción & Observaciones \\
\hline $\begin{array}{l}\text { Tipo de } \\
\text { documento }\end{array}$ & $\begin{array}{l}\text { Artículos publicados en revistas } \\
\text { científicas a texto completo. }\end{array}$ & $\begin{array}{l}\text { Se incluyen artículos } \\
\text { «in press» }\end{array}$ \\
\hline Idioma & $\begin{array}{l}\text { Inglés. } \\
\text { Español. }\end{array}$ & $\begin{array}{l}\text { Si el documento se } \\
\text { encuentra en los dos } \\
\text { idiomas se incluye la } \\
\text { versión en inglés. }\end{array}$ \\
\hline Período temporal & Enero de 2010 - Diciembre de & \\
\hline Nivel educativo & 2018 & A priori todos \\
\hline $\begin{array}{l}\text { Metodología de } \\
\text { investigación }\end{array}$ & $\begin{array}{l}\text { Estudios empíricos con métodos } \\
\text { cuantitativos y/o cualitativos y } \\
\text { artículos teóricos. }\end{array}$ \\
\hline
\end{tabular}

\subsection{Análisis y selección}

Para búsqueda de los artículos seleccionados se utilizaron las bases de datos ERIC y Dialnet. ERIC es una base de datos exclusivamente del ámbito educativo que incluye la mayor parte de referencias bibliográficas 
contenidas en Scopus y WOS, y Dialnet nos aporta la visión latinoamericana sobre esta búsqueda.

Tras lanzar una primera búsqueda con la selección de palabras clave definidas en el protocolo de búsqueda y considerando solo el término de búsqueda o la combinación de términos en el título, resumen y palabra clave del texto, se obtuvieron los siguientes resultados en la primera búsqueda: ERIC lanzó un total 506 elementos y en Dialnet 556.

En la siguiente tabla se muestra el número de artículos seleccionados finalmente de acuerdo al protocolo fijado con anterioridad.

Tabla 2

Protocolo de búsqueda en base de datos

\begin{tabular}{|c|c|c|c|}
\hline Búsquedas & Base de datos & Protocolo & Resultados \\
\hline $1^{\circ}$ búsqueda & ERIC & $\begin{array}{ll} & \text { Mobile Learning } \\
\text { - } & \text { Desde } 2010 \\
\text { - } & \text { Texto completo en ERIC } \\
\text { - } & \text { Artículo de investigación } \\
\end{array}$ & 506 artículos \\
\hline $2^{\circ}$ búsqueda & ERIC & $\begin{array}{ll}\text { - } & \text { Mobile Learning \& Teaching } \\
& \text { methods } \\
\text { - } & \text { Desde } 2010 \\
\text { - } & \text { Texto completo en ERIC } \\
\text { - } & \text { Artículo de investigación }\end{array}$ & 203 artículos \\
\hline $3^{\circ}$ búsqueda & ERIC & $\begin{array}{ll}\text { - } & \text { Mobile Learning \& Technolo- } \\
\text { gy uses in education } \\
\text { - } & \text { Desde } 2010 \\
\text { - } & \text { Texto completo en ERIC } \\
\text { - } & \text { Artículo de investigación }\end{array}$ & 247 artículos \\
\hline $4^{\circ}$ búsqueda & ERIC & $\begin{array}{ll} & \text { Mobile Learning \& Teaching } \\
\text { methods \& Technology uses } \\
\text { in education } \\
\text { - } & \text { Desde } 2010 \\
\text { - } & \text { Texto completo en Eric } \\
\text { - } & \text { Artículo de investigación }\end{array}$ & 117 artículos \\
\hline $1^{\circ}$ Búsqueda & Dialnet & $\begin{array}{ll}\text { - } & \text { Aprendizaje móvil } \\
\text { - } & \text { Artículo de revista } \\
\text { - } & \text { Desde } 2010 \\
\text { - } & \text { Texto completo } \\
\end{array}$ & 556 artículos \\
\hline $2^{\circ}$ Búsqueda & Dialnet & $\begin{array}{ll}\text { - } & \text { Aprendizaje móvil y métodos } \\
& \text { de aprendizaje } \\
\text { - } & \text { Artículo de revista } \\
\text { - } & \text { Desde } 2010 \\
\text { - } & \text { Texto completo }\end{array}$ & 71 artículos \\
\hline
\end{tabular}


Aspectos pedagógicos, tecnológicos y de interacción social del aprendizaje móvil: revisión Sistemática de Literatura

Alicia González Pérez y Maria José Sosa díaz

\begin{tabular}{|c|c|c|c|}
\hline Búsquedas & Base de datos & Protocolo & Resultados \\
\hline $3^{\circ}$ Búsqueda & Dialnet & $\begin{array}{ll}\text { - } & \text { Aprendizaje móvil, métodos } \\
& \text { de aprendizaje, TIC } \\
\text { - } & \text { Artículo de revista } \\
\text { - } & \text { Desde } 2010 \\
\text { - } & \text { Texto completo }\end{array}$ & 13 artículos \\
\hline
\end{tabular}

Al final seleccionamos 117 artículos de ERIC y 13 de Dialnet. Después de esta fase se abrió otra, en la cual se entró de lleno en el contenido de los artículos y se seleccionaron solo aquellos que nos ayudaron a responder las preguntas de investigación planteadas anteriormente.

\subsection{Síntesis y organización}

En la siguiente tabla se presentan los artículos finalmente seleccionados para la revisión sistemática considerando los siguientes datos descriptivos: el país donde se desarrolla la investigación, el tipo de metodología utilizada, la muestra y las dimensiones generales que aborda cada estudio.

Tabla 2

Identificación de la selección de artículos científicos

\begin{tabular}{|c|c|c|c|c|}
\hline $\begin{array}{l}\text { Autores } \\
\text { (año) }\end{array}$ & País & Metodología & Muestra & Dimensiones de estudio \\
\hline $\begin{array}{l}\text { (Adegbija \& } \\
\text { Bola, 2015) }\end{array}$ & Nigeria & $\begin{array}{l}\text { Cuantitativa: } \\
\text { Encuestas }\end{array}$ & $\begin{array}{c}182 \\
\text { participantes }\end{array}$ & $\begin{array}{l}\text { Actitud positiva hacia el } \\
\text { aprendizaje móvil. }\end{array}$ \\
\hline $\begin{array}{l}\text { (Althunibat, } \\
\text { 2015) }\end{array}$ & Jordania & $\begin{array}{l}\text { Cuantitativa: } \\
\text { Cuestionarios }\end{array}$ & $\begin{array}{c}250 \\
\text { participantes }\end{array}$ & $\begin{array}{l}\text { Facilidad de uso y accesi- } \\
\text { bilidad. } \\
\text { Auto-eficiencia en el apren- } \\
\text { dizaje. }\end{array}$ \\
\hline $\begin{array}{l}\text { (Alhassan, } \\
\text { 2016) }\end{array}$ & $\begin{array}{l}\text { Arabia } \\
\text { Saudí }\end{array}$ & $\begin{array}{c}\text { Mixta: } \\
\text { Cuestionarios } \\
\text { y entrevistas }\end{array}$ & $\begin{array}{c}1000 \\
\text { estudiantes }\end{array}$ & $\begin{array}{l}\text { Experiencia de los estudian- } \\
\text { tes en el uso dispositivo mó- } \\
\text { vil en el aprendizaje. } \\
\text { Posibilidad de aprender en } \\
\text { cualquier momento. } \\
\text { Considera desventajas. }\end{array}$ \\
\hline $\begin{array}{l}\text { (Al-said, } \\
\text { 2015) }\end{array}$ & $\begin{array}{l}\text { Arabia } \\
\text { Saudí }\end{array}$ & $\begin{array}{l}\text { Cuantitativa: } \\
\text { Cuestionarios }\end{array}$ & $\begin{array}{c}32 \\
\text { estudiantes }\end{array}$ & $\begin{array}{l}\text { Aumenta la eficacia de } \\
\text { comunicación. } \\
\text { La actitud de uso es positiva. } \\
\text { Estudiantes tienen altas } \\
\text { competencias de uso. }\end{array}$ \\
\hline
\end{tabular}


Aspectos pedagógicos, tecnológicos y de interacción social del aprendizaje móvil: revisión Sistemática de Literatura

Alicia González Pérez y Maria José Sosa Díaz

\begin{tabular}{|c|c|c|c|c|}
\hline $\begin{array}{l}\text { Autores } \\
\text { (año) }\end{array}$ & País & Metodología & Muestra & Dimensiones de estudio \\
\hline $\begin{array}{l}\text { (Antunes } \\
\text { \& Tenorio, } \\
\text { 2010) }\end{array}$ & Brasil & $\begin{array}{l}\text { Cualitativa: } \\
\text { Foro de dis- } \\
\quad \text { cusión }\end{array}$ & $\begin{array}{c}1328 \\
\text { participantes }\end{array}$ & $\begin{array}{l}\text { Satisfacción del modelo } \\
\text { mobile learning. } \\
\text { Entorno colaborativo inte- } \\
\text { ractivo. } \\
\text { Facilidad de uso y práctico. }\end{array}$ \\
\hline $\begin{array}{l}\text { (Bahry et } \\
\text { al., 2015) }\end{array}$ & Malasia & $\begin{array}{l}\text { Cualitativa: } \\
\text { Foros, en- } \\
\text { trevistas y } \\
\text { encuestas }\end{array}$ & $\begin{array}{l}\text { Estudio de } \\
\text { caso de una } \\
\text { asignatura }\end{array}$ & $\begin{array}{l}\text { Desarrollo de competencias } \\
\text { ciudadanas. } \\
\text { Elementos de seguridad y } \\
\text { privacidad en internet. } \\
\text { Actitud favorable de uso. }\end{array}$ \\
\hline $\begin{array}{l}\text { (Becerra, } \\
\text { 2015) }\end{array}$ & México & $\begin{array}{l}\text { Mixta: } \\
\text { Encuestas } \\
\text { Foro de dis- } \\
\text { cusión } \\
\end{array}$ & $\begin{array}{l}\text { Estudio de } \\
\text { caso de una } \\
\text { asignatura }\end{array}$ & $\begin{array}{l}\text { Competencias ciudadanas. } \\
\text { Comunicación e interac- } \\
\text { ción del alumnado. }\end{array}$ \\
\hline $\begin{array}{l}\text { (Brantes et } \\
\text { al., 2013) }\end{array}$ & Brasil & $\begin{array}{l}\text { Cuantitativa: } \\
\text { Cuestionarios }\end{array}$ & $\begin{array}{l}402 \\
\text { estudiantes }\end{array}$ & $\begin{array}{l}\text { Facilidad de uso, compati- } \\
\text { bilidad y auto-eficiencia. } \\
\text { Percepción de utilidad para } \\
\text { el aprendizaje. }\end{array}$ \\
\hline $\begin{array}{l}\text { (Brazuelo } \\
\text { \& Cacheiro, } \\
\text { 2015) }\end{array}$ & España & $\begin{array}{l}\text { Cualitativa: } \\
\text { Estudio des- } \\
\text { criptivo }\end{array}$ & $\begin{array}{c}22 \\
\text { plataformas } \\
\text { MOOC }\end{array}$ & $\begin{array}{l}\text { Necesidad de adaptar los } \\
\text { MOOC a los principios del } \\
\text { mobile learning. }\end{array}$ \\
\hline $\begin{array}{l}\text { (Broadbent } \\
\text { \& Poon, } \\
2015)\end{array}$ & $\begin{array}{l}\text { Austra- } \\
\text { lia }\end{array}$ & $\begin{array}{l}\text { Revisión Bi- } \\
\text { bliográfica }\end{array}$ & & $\begin{array}{l}\text { Aprendizaje autoregulado: } \\
\text { gestión del tiempo, esfuerzo } \\
\text { y pensamiento crítico. }\end{array}$ \\
\hline $\begin{array}{l}\text { (Cheon et } \\
\text { al., 2012) }\end{array}$ & $\begin{array}{l}\text { EEUU/ } \\
\text { Corea }\end{array}$ & $\begin{array}{l}\text { Cuantitativa: } \\
\text { Cuestionario }\end{array}$ & $\begin{array}{c}177 \\
\text { estudiantes }\end{array}$ & $\begin{array}{l}\text { Aceptación del mobile } \\
\text { learning. } \\
\text { Auto-eficacia. } \\
\text { Autonomía. } \\
\text { Utilidad y facilidad de uso. }\end{array}$ \\
\hline $\begin{array}{l}\text { (Gikas } \\
\text { \& Grant, } \\
\text { 2013) }\end{array}$ & EEUU & $\begin{array}{l}\text { Cualitativa: } \\
\text { Entrevistas }\end{array}$ & $\begin{array}{l}9 \\
9 \\
\text { participantes }\end{array}$ & $\begin{array}{l}\text { Aprendizaje informal. } \\
\text { Aprendizaje colaborativo. } \\
\text { Oportunidades de inte- } \\
\text { racción, colaboración y } \\
\text { creación de contenidos. }\end{array}$ \\
\hline $\begin{array}{l}\text { (Goh et al., } \\
\text { 2010) }\end{array}$ & $\begin{array}{l}\text { Singa- } \\
\text { pur }\end{array}$ & $\begin{array}{l}\text { Mixta } \\
\text { Cuestionario } \\
\text { entrevistas }\end{array}$ & $\begin{array}{c}94 \\
\text { estudiantes }\end{array}$ & $\begin{array}{l}\text { Beneficios del blog. Eficien- } \\
\text { cia. Colaboración. } \\
\text { Comunidad de aprendizaje. } \\
\text { Aprendizaje reflexivo. }\end{array}$ \\
\hline $\begin{array}{c}\text { (Hsu, } \\
\text { Chang, \& } \\
\text { Lee, 2013) }\end{array}$ & China & $\begin{array}{l}\text { Cualitativa: } \\
\text { Análisis de } \\
\text { contenido }\end{array}$ & 18 usuarios & $\begin{array}{l}\text { Gamificación. } \\
\text { Narrativa colaborativa. }\end{array}$ \\
\hline
\end{tabular}


Aspectos pedagógicos, tecnológicos y de interacción social del aprendizaje móvil: revisión Sistemática de Literatura

Alicia González Pérez y Maria José Sosa Díaz

\begin{tabular}{|c|c|c|c|c|}
\hline $\begin{array}{c}\text { Autores } \\
\text { (año) }\end{array}$ & País & Metodología & Muestra & Dimensiones de estudio \\
\hline $\begin{array}{l}\text { (Joan, } \\
\text { 2013) }\end{array}$ & India & $\begin{array}{l}\text { Cuantitativa: } \\
\text { Cuestionarios }\end{array}$ & $\begin{array}{l}100 \\
\text { estudiantes } \\
\text { y } 60 \\
\text { profesores }\end{array}$ & $\begin{array}{l}\text { Aprendizaje académico. } \\
\text { Los profesores usuarios de } \\
\text { móviles realizan aprendi- } \\
\text { zaje más centrado en el } \\
\text { estudiante. } \\
\text { Aumenta el rendimiento } \\
\text { académico. }\end{array}$ \\
\hline $\begin{array}{l}\text { (Kong } \\
\text { \& Song, } \\
2015)\end{array}$ & China & $\begin{array}{l}\text { Investigación } \\
\text { mixta }\end{array}$ & $\begin{array}{c}26 \\
\text { profesores }\end{array}$ & $\begin{array}{l}\text { Aprendizaje personalizado. } \\
\text { Aprendizaje profundo y } \\
\text { activo. }\end{array}$ \\
\hline $\begin{array}{l}\text { (Laine, } \\
\text { Veermans, } \\
\text { Lahti, \& } \\
\text { Veermans, } \\
\text { 2017) } \\
\end{array}$ & $\begin{array}{l}\text { Finlan- } \\
\text { dia }\end{array}$ & $\begin{array}{l}\text { Cualitati- } \\
\text { va: grupos } \\
\text { focales }\end{array}$ & $\begin{array}{l}2 \text { grupos de } \\
\quad \mathrm{N}=18 \\
\text { estudiantes }\end{array}$ & Aprendizaje móvil. \\
\hline $\begin{array}{l}\text { (Nakano } \\
\text { Osores et } \\
\text { al., 2013) }\end{array}$ & Perú & $\begin{array}{c}\text { Mixta: } \\
\text { Cuestionario, } \\
\text { Grupos de } \\
\text { discusión y } \\
\text { observacio- } \\
\text { nes } \\
\end{array}$ & $\begin{array}{l}18 \\
\text { estudiantes y } \\
3 \text { profesores }\end{array}$ & $\begin{array}{l}\text { Aumento de esfuerzo, auto- } \\
\text { eficacia e intención de uso. } \\
\text { Actitud positiva. }\end{array}$ \\
\hline $\begin{array}{l}\text { (Organista } \\
\text { \& Serrano, } \\
\text { 2011) }\end{array}$ & México & $\begin{array}{l}\text { Mixta: } \\
\text { Cuestionario } \\
\text { Grupo focal }\end{array}$ & $\begin{array}{l}\text { No se } \\
\text { identifica }\end{array}$ & $\begin{array}{l}\text { Pertenencia a una comuni- } \\
\text { dad. } \\
\text { Intercambio, colaboración y } \\
\text { comunicación. } \\
\text { Desarrollo de habilidades } \\
\text { digitales. }\end{array}$ \\
\hline $\begin{array}{l}\text { (Reychav \& } \\
\text { Wu, 2015) }\end{array}$ & EEUU & $\begin{array}{l}\text { Cualitativa: } \\
\text { Recursos au- } \\
\text { diovisuales }\end{array}$ & $\begin{array}{c}1131 \\
\text { estudiantes }\end{array}$ & $\begin{array}{l}\text { Rendimiento y satisfacción. } \\
\text { Aprendizaje colaborativo. }\end{array}$ \\
\hline $\begin{array}{l}\text { (Sanchez et } \\
\text { al, 2018) }\end{array}$ & España & $\begin{array}{l}\text { Metodología } \\
\text { de Gamifica- } \\
\text { ción: Encues- } \\
\text { ta }\end{array}$ & $\begin{array}{c}60 \\
\text { estudiantes: } \\
22 \text { prueba, } \\
38 \text { control }\end{array}$ & $\begin{array}{l}\text { Aprendizaje de forma diver- } \\
\text { tida, autónoma y ayudan- } \\
\text { do a fortalecer conceptos } \\
\text { básicos sin esfuerzo. }\end{array}$ \\
\hline $\begin{array}{l}\text { (Sarrab et } \\
\text { al., 2016) }\end{array}$ & $\begin{array}{l}\text { Oman, } \\
\text { UK, } \\
\text { USA }\end{array}$ & $\begin{array}{l}\text { Cualitativa: } \\
\text { Estudios de } \\
\text { casos }\end{array}$ & $\begin{array}{l}4 \text { estudios } \\
\text { de casos }\end{array}$ & $\begin{array}{l}\text { Satisfacción del estudiante. } \\
\text { Impacto positivo en el } \\
\text { aprendizaje. } \\
\text { Disponibilidad, flexibilidad } \\
\text { y facilidad de uso. }\end{array}$ \\
\hline
\end{tabular}


Aspectos pedagógicos, tecnológicos y de interacción social del aprendizaje móvil: revisión Sistemática de Literatura Alicia González Pérez y Maria José Sosa díaz

\begin{tabular}{|c|c|c|c|c|}
\hline $\begin{array}{c}\text { Autores } \\
\text { (año) }\end{array}$ & País & Metodología & Muestra & Dimensiones de estudio \\
\hline $\begin{array}{l}\text { (Somoano } \\
\text { \& Menén- } \\
\text { dez, 2018) }\end{array}$ & España & $\begin{array}{l}\text { Cualitativa: } \\
\text { Entrevista y } \\
\text { diario }\end{array}$ & $\begin{array}{l}18 \\
\text { estudiantes } \\
1 \text { profesora }\end{array}$ & $\begin{array}{l}\text { Capacidad organizativa } \\
\text { y de trabajo en equipo, y } \\
\text { pensamiento autónomo. } \\
\text { Motivación. }\end{array}$ \\
\hline $\begin{array}{l}\text { (Suárez et } \\
\text { al., 2013) }\end{array}$ & España & $\begin{array}{c}\text { Cualitativa: } \\
\text { Entrevistas } \\
\text { Foros de } \\
\text { discusión } \\
\end{array}$ & $\begin{array}{c}10 \\
\text { entrevistas }\end{array}$ & $\begin{array}{l}\text { Protagonismo del estudian- } \\
\text { te. } \\
\text { Participación y colabora- } \\
\text { ción. }\end{array}$ \\
\hline $\begin{array}{l}\text { (Su \& } \\
\text { Cheng, } \\
2013) \\
\end{array}$ & Taiwán & $\begin{array}{l}\text { Cuantitativa: } \\
\text { Estudio cuasi } \\
\text { experimental }\end{array}$ & - & $\begin{array}{l}\text { Gamificación. } \\
\text { Logros de aprendizaje. } \\
\text { Experiencias satisfactorias. }\end{array}$ \\
\hline $\begin{array}{l}\text { (Tabuen- } \\
\text { ca et al., } \\
2015)\end{array}$ & $\begin{array}{l}\text { Holan- } \\
\text { da }\end{array}$ & $\begin{array}{l}\text { Cualitativa: } \\
\text { Estudio longi- } \\
\text { tudinal }\end{array}$ & $\begin{array}{c}89 \\
\text { estudiantes }\end{array}$ & $\begin{array}{l}\text { Aprendizaje auto-regulado. } \\
\text { Mejora en la gestión del } \\
\text { tiempo y planificación. }\end{array}$ \\
\hline $\begin{array}{l}\text { (Wang et } \\
\text { al., 2013) }\end{array}$ & EEUU & $\begin{array}{l}\text { Cuantitativa: } \\
\text { Encuesta en } \\
\text { línea }\end{array}$ & $\begin{array}{c}256 \\
\text { estudiantes }\end{array}$ & $\begin{array}{l}\text { Aprendizaje auto-regulado, } \\
\text { auto-eficiencia del apren- } \\
\text { dizaje. } \\
\text { Motivación y satisfacción. }\end{array}$ \\
\hline $\begin{array}{l}\text { (Yot \& Mar- } \\
\text { celo, 2015) }\end{array}$ & España & $\begin{array}{l}\text { Cuantitativa: } \\
\text { Cuestionario }\end{array}$ & $\begin{array}{c}384 \\
\text { participantes }\end{array}$ & $\begin{array}{l}\text { Uso de la tecnología móvil } \\
\text { en Educación. } \\
\text { Adaptación a los hábitos } \\
\text { personales. }\end{array}$ \\
\hline $\begin{array}{l}\text { (Zaheer, } \\
\text { Butt, Ana- } \\
\text { tolyevna, } \\
\text { \& Salmani, } \\
\text { 2018) }\end{array}$ & Brasil & $\begin{array}{l}\text { Método de } \\
\text { observación } \\
\text { y recolección } \\
\text { de datos: } \\
\text { caso de estu- } \\
\text { dio múltiple } \\
\end{array}$ & $\begin{array}{l}2 \text { grupos de } \\
\text { estudiantes } \\
\text { de un } \\
\text { curso de } \\
\text { pedagogía }\end{array}$ & $\begin{array}{l}\text { Aprender haciendo. } \\
\text { Aprendizaje por objetivos. } \\
\text { Aprendizaje colaborativo. }\end{array}$ \\
\hline
\end{tabular}

\section{Resultados}

Los resultados se han organizado para dar respuesta a las preguntas que hemos planteado en la investigación. Para ello se han identificado tres dimensiones que convergen en el aprendizaje móvil: los factores pedagógicos y disciplinares, los aspectos de interacción y colaboración social, y los aspectos tecnológicos en cuanto a la usabilidad del dispositivo. 
Aspectos pedagógicos, tecnológicos y de interacción social del aprendizaje móvil: revisión Sistemática de Literatura

Alicia González Pérez y Maria José Sosa Díaz

\subsection{Factores pedagógicos a considerar en el mobile learning}

El potencial del mobile learning en contextos formales e informales es un fenómeno creciente en el campo de la investigación educativa y en la práctica de las instituciones educativas. También gana importancia la idea de que el mobile learning se refiere y se aproxima más a lo informal que a lo formal. Por ello es importante considerar el valor de éste en cuestiones como la autonomía del alumnado (González-Pérez, 2019), la auto-regulación del aprendizaje, la influencia del aprendizaje informal en el estudiante, el rendimiento académico, y la aplicación de metodologías activas de enseñanza-aprendizaje (Godejord, González-Pérez, Figueiredo, \& Solmaz, 2016).

Según algunos de los estudios revisados, la incorporación de los dispositivos móviles al proceso de enseñanza-aprendizaje favorece el desarrollo de aprendizajes más profundos y significativos que suponen una mejora en el rendimiento académico y un aumento en las calificaciones (Joan, 2013; Nakano Osores et al., 2013; Sánchez et al, 2018). Así pues, se ve aumentada la percepción de auto-eficacia en el alumnado (Cheon et al., 2012).

Sin embargo, uno de los aspectos que más se apuntan en las investigaciones revisadas es la transcendencia que tiene el aprendizaje informal cuando se usan tecnologías móviles. El desarrollo conceptual del aprendizaje informal no es algo nuevo y suele situar al estudiante como actor principal dentro del proceso de aprendizaje (Gikas \& Grant, 2013). Fundamentalmente, esto es debido a que es el aprendiz el que regula el uso del dispositivo móvil. En este sentido, se puede afirmar que las tecnologías móviles favorecen aprendizajes más autónomos (Sanchez et al, 2018), que pueden ser más o menos estructurados, y que están basados en las necesidades e inquietudes de los propios aprendices (Cheon et al., 2012). Según Wang, Shannon, \& Ross (2013) el éxito de este tipo de aprendizaje está en la implementación de metodologías activas y en el compromiso que el aprendiz adquiere en el proceso de aprendizaje. Por tanto, ser estudiante móvil implica ser más independiente en la gestión de los aprendizajes y requiere el desarrollo de habilidades de auto-control, organización y planificación (Tabuenca et al., 2015).

Broadbent \& Poon (2015) hacen una exhaustiva revisión bibliográfica sobre la relación existente entre el desarrollo de estrategias de aprendizaje auto-regulado y el nivel de adquisición de logros en cursos online, y 
afirman que hay nueve elementos clave para el éxito de éstos. Éstos son: la metacognición, la gestión del tiempo, la regulación del esfuerzo, el aprendizaje entre pares, la elaboración de la información, el ensayo, la organización, el pensamiento crítico y la búsqueda de ayuda.

Según el cognitivismo social, en el aprendizaje auto-regulado confluyen tres elementos: la auto observación, la autovaloración y la auto respuesta (Broadbent \& Poon, 2015). Lo más importante de este modelo es que el aprendizaje no es algo fijo y que puede ser modificado, con el objetivo de lograr mejores resultados académicos. Los estudiantes pueden utilizar una amplia variedad de estrategias cognitivas, metacognitivas y de gestión de recursos para autorregular sus aprendizajes (Broadbent \& Poon, 2015; Nakano Osores et al., 2013; Tabuenca et al., 2015; Wang et al., 2013).

Pero también es todo un reto permitir que las aplicaciones y recursos móviles puedan adaptarse a los diferentes ritmos de aprendizaje que tienen los alumnos (Kong \& Song, 2015; Yot \& Marcelo, 2015). Por ello, es importante que las aplicaciones móviles sean flexibles y adaptables a diferentes contextos de aprendizaje y que puedan ser utilizadas en múltiples plataformas y formatos para facilitar el acceso de todos los usuarios potenciales (Brazuelo \& Cacheiro, 2015; Sanchez et al, 2018; Sarrab et al., 2016; Yot \& Marcelo, 2015). De esta manera, el uso de dispositivos móviles para el aprendizaje puede proporcionar a las instituciones educativas oportunidades únicas para ofrecer una enseñanza más individualizada, favorecer la auto-regulación y la autonomía del alumno (Althunibat, 2015; Laine, Veermans, Lahti, \& Veermans, 2017).

La creciente adquisición de tecnologías móviles y uso de Recursos Educativos Abiertos (REA) abre nuevas líneas de trabajo centradas en la mejora de experiencias de enseñanza-aprendizaje en el ámbito de la Educación. Reaccionando a las prioridades que se marcan en el documento base "Rethinking Education: Investing in skills for better socioeconomic outcomes" (European Commission, 2012) para la Educación, formación y juventud, la Unión Europea señala la necesidad de estimular el aprendizaje abierto y flexible. De ahí que se señale la importancia de tender hacia una Educación de calidad basada en la combinación de diferentes enfoques y uso de REA, que vayan acompañados de unos estándares de calidad y unos mecanismos de evaluación y validación de las habilidades y competencias adquiridas. El mobile learning encaja perfectamente con la filosofía que está detrás del uso y utilización de 
Aspectos pedagógicos, tecnológicos y de interacción social del aprendizaje móvil: revisión Sistemática de Literatura

Alicia González Pérez y Maria José Sosa Díaz

Ios REA para favorecer nuevas ecologías de aprendizaje (Becerra, 2015).

Sin duda las posibilidades de acceso que permite el mobile learning y la accesibilidad a recursos bajo el paraguas de los REA incrementan las posibilidades educativas por diversas razones. En primer lugar, los REA son recursos que se ponen en práctica y se prestan a ser actualizados por otros docentes, lo que asegura la calidad en términos técnicos y de contenidos. En segundo lugar, el profesorado puede mejorar y adaptar el recurso a las características del alumnado, así como al contexto y a los objetivos educativos. En tercer lugar, los mismos estudiantes pueden modificar los recursos favoreciendo así el aprendizaje activo y fomentando la transferencia del conocimiento adquirido. En cuarto lugar, el estudiante siempre lo va a tener disponible gratuitamente lo que permite reducir la brecha digital (Becerra, 2015). Sin embargo, se advierte que el aprendizaje en contextos móviles y en diferentes momentos puede fragmentar el conocimiento y proporcionar esquemas de conocimiento incompletos.

Lo cierto es que ambos contextos de aprendizaje, formales e informales, han de aprovechar las potencialidades del mobile learning para introducir metodologías activas de aprendizaje, centradas en los estudiantes, y otros elementos claves para dinamizar su uso como es la gamificación (Hsu et al., 2013). Según la literatura, la gamificación es un elemento clave para animar y motivar al estudiante durante el proceso de aprendizaje ya que se pueden introducir múltiples dimensiones y mecanismos del juego, para que el alumno pueda diseñar sus propios itinerarios de aprendizaje (Sanchez et al, 2018; Su \& Cheng, 2013).

\subsection{Aspectos de interacción y colaboración social a considerar en el mobile learning}

El mobile learning no sólo supone mayores posibilidades de acceder al conocimiento sino también de generar nuevas estrategias de aprendizaje más colaborativas en las que el estudiante tenga mayor protagonismo al crear sus propias estrategias de trabajo y comunicación (Gikas \& Grant, 2013; Somoano \& Menéndez, 2018). Estudios como Suarez, Crescenzi \& Grané (2013) señalan que uno de los aspectos más valorados por los estudiantes es el aprendizaje colaborativo y la posibilidad de intercambiar experiencias de forma directa con el resto de alumnado y profesorado. De manera que Somoano \& Menéndez, 2018 señalan que el aprendiza- 
je cooperativo desarrollado es uno de los principales elementos motivacionales para el aprendizaje.

Además, Suarez, Crescenci \& Grané (2013) identifican algunas ventajas del mobile learning en el sentido que supone, un uso exponencial de las tecnologías de la web social y participativa, facilitan el acceso a los datos, la creación de contenidos textuales y audiovisuales, y el acceso desde cualquier lugar y en cualquier momento (Alhassan, 2016).

Por tanto, el mobile learning puede mejorar la interacción entre el alumnado debido a que les da la oportunidad de comunicar sus ideas por medio de mensajes de texto, correos electrónicos, participando en foros de debates, entre otras posibilidades comunicativas, lo que conduce a una mayor implicación y participación en las actividades de aprendizaje (Brantes et al., 2013; Organista \& Serrano, 2011). Además, el uso de tecnologías móviles permite a los usuarios utilizar una gama de recursos más amplia en la que se incluyen textos, imágenes, audios, vídeos, e-books, artículos electrónicos, noticias en línea, blogs, aplicaciones móviles, videojuegos (Brantes et al., 2013), y posibilita el intercambio de ideas, información, experiencias, y buenas prácticas (Gikas \& Grant, 2013; Sarrab et al., 2016; Somoano \& Menéndez, 2018).

Incluso, Becerra (2015) afirma que el mobile learning favorece la comunicación y la colaboración entre el alumnado de tal forma que existen indicios de que el uso de dispositivos móviles en las actividades académicas estimula el desarrollo de la competencia cívica y ciudadana, una actitud crítica, respeto a la diversidad y responsabilidad social por la búsqueda del bien común.

Reychav \& Wu (2015) destacan que los dispositivos móviles pueden mejorar la comunicación, la interacción y la colaboración entre los distintos agentes implicados en el proceso de enseñanza-aprendizaje, ofrecer más oportunidades de aprendizaje para personas y grupos geográficamente dispersos, estimular el aprendizaje activo, facilitar el acceso al contenido y reducir el tiempo dedicado a la tarea.

Otro elemento clave es el tiempo de interacción que se establece entre el alumnado, profesorado y la propia tecnología con el fin de construir una actitud positiva hacia el aprendizaje y favorecer la satisfacción del alumno (Antunes \& Tenorio, 2010).

Por un lado, los estudiantes de hoy esperan que la interacción con el docente sea rápida, por lo que cualquier retraso en responder las solicitudes de ayuda o comentarios deben evitarse (Sarrab et al., 2016). Por 
Aspectos pedagógicos, tecnológicos y de interacción social del aprendizaje móvil: revisión Sistemática de Literatura

Alicia González Pérez y Maria José Sosa Díaz

otro lado, el alumnado necesita que las herramientas para la interacción con los demás compañeros sean rápidas y adaptadas a sus necesidades, de forma que éstos se sientan cómodos al utilizarlas y puedan comunicarse siempre que lo consideren oportuno, favoreciendo así, la construcción de nuevos conocimientos y significados (Gikas \& Grant, 2013; Sarrab et al., 2016). Es por este motivo, que la comunicación instantánea es un elemento clave en el mobile learning debido a que permite que la interacción entre usuarios sea más ágil (Sarrab, et al., 2016).

Además, la integración y uso de redes sociales y blogs en el mobile learning puede facilitar la comunicación inmediata y proporcionar al estudiante el sentido de pertenencia e integración en una comunidad de aprendizaje (Goh et al., 2010; Organista \& Serrano, 2011).

En términos de alfabetización digital, el paradigma de cultura participativa está centrado en la expresión individual de cada uno en la comunidad que nos envuelve centrándonos en elementos claves como la apropiación, simulación, multitarea, inteligencia colectiva, el uso de los transmedia, el trabajo en red y la negociación.

\subsection{Aspectos tecnológicos a considerar en el mobile learning}

Lo cierto es que la característica móvil del mobile learning tiene más que ver con la movilidad del usuario que lleva el dispositivo móvil (Smartphone, Tablet, laptop) que con el propio dispositivo. Es decir, que la movilidad se produce cuando el usuario cambia continuamente de contexto, y es en ese cambio de contextos donde se usa una tecnología móvil. Aclarar esta cuestión es importante, pues debido a ello emergen otros conceptos clave como son la ubicuidad, portabilidad, geolocalización, entre otros (Adegbija \& Bola, 2015; Al-said, 2015; Bahry et al., 2015; Nakano Osores et al., 2013). Según Zaheer, Butt, Anatolyevna, \& Salmani (2018) el aprendizaje móvil favorece la innovación y desafíos en su implementación, que se enfocan en la conectividad, portabilidad, flexibilidad, la autonomía de los estudiantes y nuevas formas de comunicación.

Así pues, algunos de los aspectos técnicos que favorecen la calidad del mobile learning son: la rápida respuesta, flexibilidad, adaptabilidad, facilidad de uso, facilidad de mantenimiento, funcionalidad, fiabilidad, conectividad, el rendimiento, el interfaz del usuario y la seguridad (Bahry et al., 2015; Brantes et al., 2013; Sarrab et al., 2016). 
Hablar de aprendizaje ubicuo, en contextos formales e informales, nos lleva a pensar en modelos de aprendizaje diferentes. Lo cierto es que integrar el mobile learning en la comunidad educativa genera cierta resistencia debido, fundamentalmente, a que los dispositivos móviles tienen pantallas demasiado pequeñas para permitir el aprendizaje, suponen una distracción, son una opción cara y se requiere capacidad de almacenamiento. La reducida pantalla de las tecnologías móviles puede ocasionar problemas en la visualización del contenido, así como en la limitación del texto que se puede mostrar. Esto conlleva a que los teclados también sean reducidos y no existan las funciones del ratón, lo que ralentiza el desarrollo de la tarea y dificulta la construcción de documentos (Organista \& Serrano, 2011). Por tanto, se considera que los smartphones y las tabletas son más intuitivos, pero menos prácticos para trabajar.

Sin embargo, el uso de tecnologías móviles favorece la realización de diferentes tareas al mismo tiempo (Chen \& Yan, 2016). Es decir, mientras en una tecnología se busca un contenido, en otra se puede comunicar uno con otros espacios y personas, ver un vídeo, una fotografía, etc. En este sentido, algunos autores apuntan que el uso de tecnologías móviles puede distraer e ir en perjuicio del aprendizaje (Chen \& Yan, 2016). No obstante, Somoano \& Menéndez (2018) concluyen en su investigación que utilizar herramientas digitales en la práctica educativa, como son las aplicaciones móviles, promueve en el estudiante aprendizajes más auténticos para el estudiante y más adaptados al contexto cultural y social de éste.

\section{Conclusiones y discusión}

Las limitaciones del modelo tradicional de escuela han inspirado cambios en cómo la educación debe abordar los desafíos actuales y futuros. Actualmente, la emergencia de tecnologías móviles (smartphones, laptops, tablets, consolas de videojuegos, lectores electrónicos, tecnologías de asistencia para aprendices con diversidad funcional, calculadoras digitales, etc.) está directamente relacionada con pautas y hábitos de consumo que nos llevan a considerar los dispositivos móviles como medios principales de comunicación, información, entretenimiento y aprendizaje. El aprendizaje móvil conecta claramente con experiencias 
Aspectos pedagógicos, tecnológicos y de interacción social del aprendizaje móvil: revisión Sistemática de Literatura

Alicia González Pérez y Maria José Sosa Díaz

de aprendizaje situadas en aspectos claves de la vida diaria, del trabajo, y no solamente centradas en la escuela, entendida como institución educativa formal. Esto crea tensiones entre lo estrictamente educativo y reglado, y los intereses y necesidades personales de los estudiantes en relación con diversas situaciones, experiencias, vivencias y contextos en los que se desarrolla y crece el estudiante. Ya Fombona \& Pascual (2013) destacaban los beneficios que generaban los dispositivos móviles en las actividades educativas en temas como la comunicación, la economía del tiempo y la ubiquidad.

Esta revisión sistemática de literatura muestra evidencias y elementos clave que ayudan a entender la importancia del aprendizaje móvil para diseñar un proceso de aprendizaje centrado en el alumno (Broadbent \& Poon, 2015) y que atienda las necesidades de colaboración e interrelación entre usuarios en entornos sociales online y fuera del aula. Esto es debido a que el aprendizaje móvil incluye elementos de la propia tecnología que engancha y anima a los usuarios portadores de la misma a aumentar el grado de funcionalidad, convergencia multimedia, ubiquidad, conectividad y geolocalización, en su relación con el aprendizaje fuera del aula.

Nuevos medios tecnológicos convergen con prácticas culturales que transforman el aprendizaje y su efecto fuera de las instituciones formales. Las tecnologías móviles amplían la cobertura de uso y favorecen nuevos contextos de aprendizaje donde las prácticas educativas también sufren modificaciones (Gikas \& Grant, 2013). Es por ello que los dispositivos móviles con conexión a Internet dejan de ser una tecnología más para convertirse en un medio de comunicación fundamental para la gestión de la información, la consulta de contenidos, el aprendizaje informal, la incorporación de metodologías activas de aprendizaje centradas en el alumno (Gikas \& Grant, 2013; González-Pérez, 2019) y la autoregulación del aprendizaje (Wang, Shannon \& Ross, 2013; Broadbent \& Poon, 2015), la producción multimedia, la gamificación (Hsu et al., 2013) y la interacción con otros usuarios (Bahry et al, 2015). No se necesita más de lo que se puede llevar en el bolsillo para resolver un problema y estar conectado con una comunidad de usuarios.

Repensar la educación que queremos para una sociedad cada vez más digitalizada es fundamental cuando se observa que la tecnología móvil no solo afecta a qué necesitaremos para aprender, sino cómo aprenderemos en un futuro. La clave para preparar adecuadamente a las 
generaciones futuras en el mundo digital está en rediseñar la formación hacia la participación y la colaboración digital (Organista \& Serrano, 2011), en y más allá de la propia educación formal. Por tanto, algunos de los principios en la Educación del futuro serán el aprendizaje autónomo, aprendizaje centrado en las necesidades del alumno, aprendizaje en red, competencia ciudadana (Becerra, 2015), aprendizaje móvil, y la reputación digital. La pedagogía utilizará métodos centrados en la generación de conocimiento y fuentes abiertas para el aprendizaje. Y las instituciones educativas se caracterizarán por ser estructuras más horizontales y flexibles, que darán más importancia a la necesidad de trabajar en red.

Así pues, se pueden predecir algunos cambios en la educación del futuro, que van en la línea de ofrecer un aprendizaje más personalizado, colaborativo e informal (González-Pérez, 2019). Por ello, es importante contemplar los ritmos de aprendizaje de los alumnos, las estrategias y estilos de aprendizaje personalizados, la búsqueda de colaboración intercentros, la innovación en la práctica educativa, el desarrollo de la creatividad, la participación social y la puesta en práctica de estructuras para la orientación y apoyo pedagógico en red. El aprendizaje permanente obliga a los estudiantes, a que ellos mismos asuman la responsabilidad de aprender. Y el aprendizaje móvil facilita dinámicas de colaboración entre lo que se aprende en el aula y en otros lugares, conecta a estudiantes de forma remota favoreciendo oportunidades de intercambiar información, facilita la creación de conocimiento más auténtico, y combina nuevas experiencias de aprendizaje donde cabe lo formal y lo informal. Aunque estos términos no son nuevos en la jerga pedagógica si serán parte del paradigma de aprendizaje que caracterizara a las sociedades digitalizadas.

Según el Plan europeo para el desarrollo de la competencia digital y el desarrollo tecnológico en la educación, tres son las prioridades para enfrentar los desafíos y oportunidades de la educación en la era digital: hacer un mejor uso de la tecnología digital para la enseñanza y aprendizaje, desarrollar las competencias y habilidades digitales, y mejorar la educación a través de un mejor análisis y previsión de datos. Este es el reto que tiene el profesorado del siglo XXI para favorecer el aprendizaje móvil en las distintas etapas educativas.

Por tanto, la reflexión sobre el impacto del aprendizaje móvil en las escuelas para la calidad educativa y la inclusión social no debe considerarse como algo liviano. Hay que darle el carácter necesario para que las 
Aspectos pedagógicos, tecnológicos y de interacción social del aprendizaje móvil: revisión Sistemática de Literatura

Alicia González Pérez y Maria José Sosa Díaz

escuelas y colegios no prohíban el uso de los móviles en sus instalaciones, obligando en muchos casos a que los maestros y profesores lo usen de forma clandestina. Es necesario que tanto las políticas institucionales, los padres, los alumnos y los profesores se muestren más abiertos a pensar cómo estas herramientas pueden usarse de forma responsable para favorecer los procesos de enseñanza-aprendizaje de forma sostenible en el tiempo. También es importante apuntar que una de las limitaciones de este estudio es que no se ha tenido en cuenta la importancia de sensibilizar y concienciar, a estudiantes, padres y profesores y sociedad en general, sobre los riesgos de ciberseguridad a los que se enfrentan con la alfabetización mediática.

\section{Referencias}

Adegbija, M. V. \& Bola, O. O. (2015). Perception of undergraduates on the adoption of mobile technologies for learning in selected universities in Kwara state, Nigeria. Procedia - Social and Behavioral Sciences, 176, 352-356. http://doi.org/10.1016/j. sbspro.2015.01.482

Alhassan, R. (2016). Mobile Learning as a Method of Ubiquitous Learning: Students' Attitudes, Readiness, and Possible Barriers to Implementation in Higher Education. Journal of Education and Learning, 5(1), 176-189. http://doi.org/10.5539/jel.v5n1p176

Al-said, K. M. (2015). Students' Perceptions of Edmodo and Mobile Learning and their Real Barriers towards them. TOJET: The Turkish Online Journal of Educational Technology, 14(2), 167-180.

Althunibat, A. (2015). Determining the factors influencing students' intention to use m-learning in Jordan higher education. Computers in Human Behavior, 52, 65-71. http://doi.org/10.1016/j.chb.2015.05.046

Antunes, O. \& Tenorio, L. R. (2010). Student Perspectives about Mobile Learning Initiatives at Open University of Brazil: The Mobile Phone Issue. Acta Scientiarum. Education, 32(2), 219-223. http://doi.org/10.4025/actascieduc.v32i2.11545

Bahry, F. D. S., Anwar, N., Amran, N., \& Rias, R. P. M. (2015). Conceptualizing Security Measures on Mobile Learning for Malaysian Higher Education Institutions. Procedia - Social and Behavioral Sciences, 176, 1083-1088. http://doi.org/10.1016/j.sbspro.2015.01.582

Becerra, M. L. (2015). Desarrollo de competencias ciudadanas: una experiencia desde el m-learning en la formación de antropólogos. Aularia: Revista Digital de Comunicación, 4(2), 23-32.

Brantes, J., Sabino, A., Azevedo, M. L., Campos, H., Calvão, A. M., \& Junqueria, C. (2013). Intention to Use M-Learning in Higher Education Settings. Pretexto, 15, 11-28.

Brazuelo, F., \& Cacheiro, M. L. (2015). Estudio de adaptabilidad para dispositivos móviles en plataformas MOOC. RED-Revista de Educación a Distancia, 47, 1-13. 
Aspectos pedagógicos, tecnológicos y de interacción social del aprendizaje móvil: revisión Sistemática de Literatura Alicia González Pérez y Maria José Sosa díaz

Broadbent, J., \& Poon, W. L. (2015). Self-regulated learning strategies \& academic achievement in online higher education learning environments: A systematic review. Internet and Higher Education, 27, 1-13.

Chen, Q., \& Yan, Z. (2016). Does multitasking with mobile phones affect learning? A review. Computers in Human Behavior, 54, 34-42. http://doi.org/10.1016/j. chb.2015.07.047

Cheon, J., Lee, S., Crooks, S. M., \& Song, J. (2012). An investigation of mobile learning readiness in higher education based on the theory of planned behavior. Computers \& Education, 59(3), 1054-1064. http://doi.org/10.1016/j.compedu.2012.04.015

European Commission (2012). Communication from the Commission to the European Parliament, the Council, the European Economic and Social Committee and the Committee of the regions. Rethinking Education: Investing in skills for better socioeconomic outcomes. COM (2012) 669 final.

Fombona, J. \& Pascual Ma A. (2013). Beneficios del m-learning en la Educación Superior. Educatio Siglo XXI, 31(2), 211-234.

Gikas, J., \& Grant, M. M. (2013). Mobile computing devices in higher education: Student perspectives on learning with cellphones, smartphones \& social media. Internet and Higher Education, 19, 18-26. http://doi.org/10.1016/j.iheduc.2013.06.002

Godejord, B. J., González-Pérez, A., Figueiredo, M., \& Solmaz, A. (2016) Breaking free of the classroom: implementing digital media to enhance students' involvement in learning mathematics, ICERI2016 Proceedings, Seville, 14th-16th November, 2016, pp. 4144-4150.

Goh., J.W.P., Quek, C. J., \& Lee, O. K. (2010). An Investigation of Students' Perceptions ofLearning Benefits of Weblogs in an East Asian Context: A Rasch Analysis. Educational Technology \& Society, 13(2), 90-101.

Gómez, P., \& Monge, C. (2013). Potencialidades del teléfono móvil como recurso innovador en el aula: una revisión teórica. Didáctica, Innovación Y Multimedia, 9(26), $1-16$.

Gómez, E., Fernando, D., Aponte, G., \& Betancourt, L. A. (2014). Metodología para la revisión bibliográfica y la gestión de información de temas científicos, a través de su estructuración y sistematización. DYNA, 81(184), 158-163.

González-Pérez, A. (2019). Marco pedagógico que sustenta el diseño de la aplicación MILAGE Aprender+ para aprender matemáticas en las escuelas secundarias. En F.I. Revuelta \& I. Pedrera (Ed), Retos y evidencias en la investigación con videojuegos en educación (pp. 117-128). Barcelona: Octaedro.

Guirao, J. A., Olmedo, A., \& Ferrer, E. (2008). El artículo de revisión. Revista Ibero-Americana de Enfermería Comunitaria, 1(6), 1-25.

Hsu, S. H., Chang, J. W., \& Lee, C. C. (2013). Designing attractive gamification features for collaborative storytelling websites. Cyberpsychology, Behavior, and Social Networking, 16(6), 428-435.

Joan, R. R. (2013). Research papers a study on mobile learning as a learning style. I-Manager's Journal on School Technology, 8(4), 29-37.

Kitchenham, B. (2004). Procedures for Performing Systematic Reviews. UK and Autralia: Joint Technical Report. Retrieved from http://www.inf.ufsc.br/ aldo.vw/kitchenham.pdf 
Aspectos pedagógicos, tecnológicos y de interacción social del aprendizaje móvil: revisión Sistemática de Literatura

Alicia González Pérez y Maria José Sosa Díaz

Kong, S. C. \& Song, Y. (2015). An Experience of Personalized Learning Hub Initiative Embedding BYOD for Reflective Engagement in Higher Education. Computers \& Education, 88, 227-240. http://doi.org/10.1016/j.compedu.2015.06.003

Koole, M. L. (2009) 'A Model for Framing Mobile Learning', in Ally, Mohamed. (ed.), Mobile Learning: Transforming the Delivery of Education and Training, (pp. 25-50), Athabasca University, Canada: Edmonton.

Laine, E., Veermans, M., Lahti, A., \& Veermans, K. (2017). Generation of Student Interest in an Inquiry-Based Mobile Learning Environment. Frontline Learning Research, 5(4), 42-60. https://doi.org/10.14786/flr.v5i4.306

Mishra, P., \& Koehler, M. J. (2006). Technological pedagogical content knowledge: A framework for teacher knowledge. The Teachers College Record, 108(6), 1017-1054.

Nakano, T., Mija, A., Begazo, J., Garret, P., Velasco, A., \& Rosales, A. M. (2013). Uso de tablets en la Educación superior: una experiencia con iPads. Digital Education Review, 24, 135-161.

Organista, J., \& Serrano, A. (2011). Implementación de una actividad educativa en modo colaborativo con apoyo de smarthpones: una experiencia universitaria. EDUTEC: Revista Electrónica de Tecnología Educativa, 36, 1-19.

Rannu, R., Saksing, S., \& Mahlakõiv, T. (2010). Mobile government: 2010 and beyond. White paper, Mobi Solutions Ltd.

Reychav, I. \& Wu, D. (2015). Mobile collaborative learning: The role of individual learning in groups through text and video content delivery in tablets. Computers in $\mathrm{Hu}$ man Behavior, 50, 520-534. http://doi.org/10.1016/j.chb.2015.04.019

Sarrab, M.; Elbasir, M., \& Alnaeli, S. (2016). Towards a quality model of technical aspects for mobile learning services : An empirical investigation. Computers in Human Behavior, 55, 100-112. http://doi.org/10.1016/j.chb.2015.09.003

Somoano, Y. \& Menéndez, J. I. (2018). Percepciones de alumnado y profesorado sobre una intervención de mobile learning en Inglés como Lengua Extranjera. Innoeduca: international journal of technology and educational innovation, 4(1), 79-87. http://doi. org/10.24310/innoeduca.2018.v4i1.3024

Soykan, E., \& Uzunboylu, H. (2015). The review of published articles on mobile learning area in EBSCO database. Procedia - Social and Behavioral Sciences, 182, 710-717. http://doi.org/10.1016/j.sbspro.2015.04.818

Su, C. H., \& Cheng, C. H. (2013). A mobile game-based insect learning system for improving the learning. Procedia - Social and Behavioral Sciences, 103, 42-50. http://doi. org/10.1016/j.sbspro.2013.10.305

Suárez, R., Crescenzi, L., \& Grané, M. (2013). Análisis del entorno colaborativo creado para una experiencia de mobile learning. Education in the Knowledge Society (EKS), 14(1), 101-122.

Tabuenca, B., Kalz, M., Drachsler, H., \& Specht, M. (2015). Time will tell: The role of mobile learning analytics in self-regulated learning. Computers \& Education, 89, 53-74. http://doi.org/10.1016/j.compedu.2015.08.004

Tedesco, J. C. (2000). Educar en la sociedad del conocimiento. Buenos Aires: Fondo de Cultura Económica de Argentina.

Wang, C. H., Shannon, D., \& Ross, M. (2013). Students' characteristics, self-regulated 
Aspectos pedagógicos, tecnológicos y de interacción social del aprendizaje móvil: revisión Sistemática de Literatura Alicia González Pérez y Maria José Sosa Díaz

learning, technology self-efficacy, and course outcomes in online learning. Distance Education, 34, 3, 302-323. https://doi.org/10.1080/01587919.2013.835779

Yot, C. \& Marcelo, C. (2015). ¿Despega el M-Learning? Análisis de la disposición y hábitos de los usuarios. Pixel-Bit. Revista de Medios y Educación, 46, 205-218. http://doi. org/http://dx.doi.org/10.12795/pixelbit.2015.i46.13

Zaheer, S., Butt, S. M., Anatolyevna, G. V., \& Salmani, H. (2018). Do Mobile Technology in the Classroom Really Improve Learning Outcomes?. International Journal of Evaluation and Research in Education, 7(3), 188-193. https://doi.org/10.11591/ijere. v7.i3.pp188-193

\section{Notas y/o Agradecimientos*}

Este trabajo de investigación forma parte del Proyecto MILAGE - Interactive Mathematics by Implementing a Blended-learning Model with Augmented Reality and Game Book - No 2015-1-PT01-KA201-012921, financiado por la Unión Europea y dentro del Programa Erasmus Plus: Acción KA2 - Cooperación para la Innovación y el Intercambio de Buenas Prácticas - de tres años de duración (2015-2018). 
